\title{
Gastos catastróficos de salud en los hogares del Paraguay
}

\author{
Edgar Giménez ${ }^{1,2}$, Laura Flores ${ }^{2}$, José Carlos Rodríguez ${ }^{2}$, Gladys Ocampos ${ }^{3}$, Néstor Peralta ${ }^{2}$ \\ ${ }^{1}$ Universidad Nacional de Concepción, Facultad de Medicina. Paraguay \\ ${ }^{2}$ Investigación para el Desarrollo. Paraguay \\ ${ }^{3}$ Dirección General de Estadística, Encuestas y Censos (DGEEC). Paraguay \\ Cómo referenciar este artículo/
How to reference this article: \\ Edgar Giménez, Laura Flores, José Carlos
Rodríguez, Gladys Ocampos, Néstor Peralta.
Gastos catastróficos de salud en los hogares del
Paraguay.Mem. Inst. Investig. Cienc. Salud.
$2018 ; 16(2): 38-48$
}

\section{RE S U M E N}

Cada año numerosas familias enfrentan gastos excesivos ante una enfermedad a pesar de que el sistema de salud tiene como uno de sus objetivos la protección financiera de la población de forma equitativa. El objetivo que nos propusimos fue describir la incidencia del gasto catastrófico de salud en los hogares paraguayos. Como material y método analizamos el módulo de salud de la Encuesta Permanente de Hogares de la Dirección General de Estadísticas Encuestas y Censos de Paraguay del 2014. Incluimos como gasto de bolsillo los pagos directos por consultas, medicamentos, estudios de diagnóstico y hospitalizaciones de las personas enfermas o accidentadas. La capacidad de pago la definimos como la suma de todos los ingresos menos los gastos de subsistencia, utilizando una estructura conocida de gastos. Calculamos el gasto catastrófico de salud utilizando distintos umbrales de relación entre el gasto de bolsillo con la capacidad de pago de los hogares (GBS/CPH). Llegamos a los siguientes resultados: En los hogares con al menos un enfermo y que tuvieron gasto de bolsillo para recibir atención la relación GBS/CPH fue en promedio de $9,1 \%$. Entre todos los hogares, las incidencias estimadas de gasto catastrófico fueron $2 \%, 2,8 \%, 4,4 \%$ y $8,7 \%$ utilizando umbrales GBS/GCS $\geq 40 \%, 30 \%, 20 \%$ y $10 \%$ respectivamente. Como conclusión, la $\mathrm{EPH}$ es un instrumento útil, pero con limitaciones, para estimar los gastos catastróficos en el sistema de salud paraguayo. Los resultados indican que es necesario diseñar e implementar mecanismos específicos para evitar este tipo de gastos.

Palabras clave: gasto catastrófico en salud, gasto de bolsillo en salud, protección financiera, Paraguay

\section{Catastrophic health expenses in households of Paraguay}

\section{A B S T R A C T}

Every year, many families face excessive expenses in the presence of an illness, despite the fact that the health system has the equitable financial protection of the population as one of its objectives. The objective of this study was to describe the incidence of catastrophic health expenses in Paraguayan households. We analyzed the health module of the Permanent Household Survey (PHS) of the General Directorate of Statistics, Surveys and Census of Paraguay of 2014. We included consultations, medicines, diagnostic studies and hospitalizations of sick or injured people as direct out-of-pocket expenses. The ability to pay is defined as the sum of all incomes minus subsistence expenses, using a known structure of expenses. We calculated the catastrophic health expenses using different thresholds for the relationship between out-of-pocket cost and household payment capacity (OPC/HPC). In households with at least one patient who had out-of-pocket cost to receive care, the OPC/HPC ratio was $9.1 \%$ in average. Among all households, the estimated incidences of catastrophic expenses were $2 \%, 2.8 \%, 4.4 \%$ and $8.7 \%$ using $40 \%, 30 \%, 20 \%$ and $10 \%$ OPC/HPC thresholds respectively. The PHS is a useful instrument, but with limitations, to estimate catastrophic expenses in the Paraguayan health system. These results indicate 
that it is necessary to design and implement specific mechanisms to avoid this type of expenses.

Keywords: catastrophic health expenses, out-of-pocket health cost, financial protection, Paraguay.

\section{INTRODUCCIÓN}

Desde la perspectiva de salud pública el análisis del gasto catastrófico en salud se ubica en los sistemas de salud, más específicamente en el modelo de financiamiento. Su relevancia radica en la comprobación empírica que las barreras económicas de accesibilidad generan exclusión ${ }^{(1)}$ y que, ante una enfermedad, los pagos directos para recibir atención constituyen arreglos inequitativos ${ }^{(2)}$. En algunos casos, puede afectar a los hogares de tal modo que pueden producir una quiebra financiera y empujarlos por debajo de la línea de pobreza ${ }^{(3)}$. Es por esto que un objetivo intrínseco del sistema de salud es brindar protección financiera a la población mediante un modelo de financiamiento equitativo y justo ${ }^{(4)}$. Para ello se diseñan e implementan diversos programas y políticas que además de responder a necesidades específicas para mejorar la salud de población buscan un mejor desempeño en los modelos de atención, de gestión y financiamiento ${ }^{(5)}$.

Cuando el sistema de salud enfrenta déficits de oferta, segmentación, fragmentación, exclusión, baja inversión pública estas intervenciones priorizan el incremento del acceso y la cobertura mediante. Una estrategia recurrente ha sido la Atención Primaria de Salud $(\mathrm{APS})^{(6,7)}$.

Las recomendaciones de políticas en la última década han puesto énfasis en: a) incluir el enfoque de los determinantes sociales de la salud (DSS) para corregir desigualdades y lograr equidad ${ }^{(8)}$, b) transformar el modelo de atención centrado en hospitales y enfermedades a otro centrado en las personas con servicios integrales ${ }^{(9)}$, c) gestionar la atención mediante redes integradas de servicios de salud (RISS) ${ }^{(10)}$, d) Incrementar el financiamiento público con un modelo que agrupe ("mancomune") fondos para compartir los riesgos y dar cobertura a una gran cantidad de población ${ }^{(3)}$, e) promover la investigación, innovación y evaluación de tecnologías sanitarias ${ }^{(11)}$ y f) incorporar la salud en todas las políticas $^{(12)}$.

En el marco de los Objetivos de Desarrollo Sostenible (ODS) de las Naciones Unidas, el desafío para la salud pública es lograr salud y bienestar para todas las personas en todas las edades en el 2013?. El reto es alcanzar la cobertura universal de salud (CUS), con recursos suficientes, para dar una respuesta efectiva a los problemas de salud reproductiva, disminuir la mortalidad materna y de menores de 5 años, poner fin a las epidemias de VIH, tuberculosis y malaria, combatir la hepatitis b y las enfermedades tropicales desatendidas, reducir las muertes prematuras causadas por enfermedades crónicas no trasmisibles (ECNT) y por las lesiones causadas por accidentes de tránsito, controlar las adicciones, el consumo excesivo de alcohol y promover la salud mental ${ }^{(13)}$.

La directora de la OMS ha señalado que la CUS es la idea más poderosa que hoy tiene la salud pública(14). Abarca tres dimensiones de cobertura: poblacional, de servicios y financiera ${ }^{(2,3)}$. Puesto que la protección financiera de la población contra gastos excesivos es parte de la cobertura financiera, la caracterización de la situación y los progresos son indicativos de las brechas y avances hacia el cumplimiento de esta meta.

Con esta ubicuidad y relevancia pasaremos a desarrollar algunos conceptos claves considerados para este trabajo.

El grado de protección financiera que tiene la población puede determinarse desde dos perspectivas: a) el modelo de financiamiento del sistema de salud y b) los pagos que realiza la población para recibir servicios de salud ante una enfermedad.

Desde la perspectiva del sistema se caracteriza el gasto total, su estructura, su composición, sus fuentes de financiamiento y su relación con la población y con el producto interno bruto (PIB), tal como se describe en las cuentas nacionales de salud ${ }^{(15)}$. Habrá mayor protección financiera cuanto mayor es la participación de fondos mancomunados de fuentes públicas y menor participación de fuentes privadas, sobre todo de los pagos directos al momento de utilizar servicios de salud. La OMS ha señalado que con una inversión de salud entre 5 y $6 \%$ del PIB se puede lograr que toda la población acceda a un conjunto de servicios y que es posible reducir al mínimo los gastos catastróficos si los pagos directos o gasto de bolsillo se reducen a 15 o $20 \%$ del gasto total ${ }^{(3)}$. 
Desde la perspectiva de los pagos realizados en el proceso de utilización de servicios de salud se busca caracterizar el gasto de bolsillo de salud (GBS), es decir, los gastos en concepto de honorarios médicos, servicios de diagnósticos, medicamentos, procedimientos terapéuticos, hospitalizaciones, dispositivos médicos u otros que están directamente relacionados con la atención médica ante una enfermedad o lesión. Se considera gasto catastrófico de salud (GCS) cuando el GBS supera un límite de capacidad de pago de los hogares. Cuando los hogares ya están en una situación financiera precaria, como en el caso de los pobres, se utiliza el concepto de gastos empobrecedores de salud (GES) ${ }^{(16)}$.

El umbral más utilizado para determinar GCS es el propuesto por Xu para la OMS, que lo definió como GBS que representan $40 \%$ o más de la capacidad de pago de los hogares $(17-20)$, pero también se utilizan con frecuencia otros umbrales como $50 \%$ y $60 \%(21), 30 \%{ }^{(22-24)}$ y $20 \%$ de la capacidad de pago ${ }^{(25,26)}$ o también como una proporción del ingreso total $(27,28)$ o sobre los gastos totales del hogar en un periodo de tiempo ${ }^{(29)}$.

Un estudio de la CEPAL del 2008 sobre siete países de América Latina que utilizó el umbral de $40 \%$ sobre la capacidad de pago, determinó que el GCS era más alto para la Argentina con $5,6 \%$ de hogares que incurrieron en gastos catastróficos, mientras que para Chile y México había afectado al $2 \%$ de los hogares, a 1,6\% de los hogares de Ecuador, al $0,6 \%$ de los hogares de Colombia y al $0,5 \%$ de los hogares en Uruguay. Utilizando el mismo umbral, en el 2011 se publicó que el $2 \%$ de los hogares brasileños tuvieron gastos catastróficos en salud?. Igualmente, en el 2017 se publicó que el 3\% de los hogares argentinos tuvieron gastos catastróficos de salud ${ }^{(30)}$.

Con umbral de 30\%, se reportó en 2013 que el 3,6\% de los hogares chilenos tuvieron gastos catastróficos ${ }^{(27)}$. Con el mismo umbral se publicó en 2015 que el $3,8 \%$ de los hogares mexicanos fueron afectados por este tipo de gastos ${ }^{(22)}$ y luego, en el 2017, otro estudio reportó que estaban afectados $2,5 \%$ de los hogares ${ }^{(31)}$.

En Colombia utilizaron como umbral el $20 \%$ de la capacidad de pago y con esta definición reportaron que el $4,1 \%$ de todos los hogares habían incurrido en gastos catastróficos (25).

En Paraguay no se han reportado estudios sobre gastos catastróficos de salud, al menos siguiendo la metodología utilizada por la OMS.

Recientemente, un estudio que analizó la Encuesta Permanente de Hogares (EPH) del 2014 reportó que $63,2 \%$ de los paraguayos tienen un riesgo de sufrir gastos catastróficos ante una atención quirúrgica, considerando el promedio pagado y el promedio de ingreso de las personas. Comparando el promedio de gastos en salud de los últimos 90 días, con el promedio de ingresos trimestrales concluyeron que los gastos en salud representan el $4,1 \%$ de los ingresos, afectando más a los más pobres, ya que para el primer quintil esta proporción se elevó a $10,4 \%$ del ingreso ${ }^{(32)}$.

Estos datos hay que analizarlos en un contexto donde el financiamiento del sistema de salud es predominantemente privado ${ }^{(33)}$. En efecto, considerando el gasto total, el gasto de bolsillo representa el 49,3\%, los pagos por seguros de medicina pre paga el 4,6\%, los recursos de la asistencia para el desarrollo $0,6 \%$ y el gasto público $45,6 \%{ }^{(34)}$. Estas características del financiamiento se dan en un sistema de salud segmentado y fragmentado ${ }^{(35)}$, con apenas $20,84 \%$ de la población cubierta por la seguridad social, $8,49 \%$ por otro tipo de seguros y $70,66 \%$ sin ningún tipo de seguro médico. Las personas que habitan zonas rurales y las que se encuentran en situación de pobreza son las que tienen menor cobertura de seguros médicos ${ }^{(36)}$.

En una publicación previa, reportamos que en Paraguay el promedio del GBS representa el $22,8 \%$ del ingreso promedio mensual. Cuando analizamos por componente resultó que el promedio de gastos por consultas fue $7,1 \%$ del ingreso promedio, $12,6 \%$ en el caso de medicamentos, $26,5 \%$ en relación a estudios diagnósticos y que ante una internación el gasto promedio fue el $193,1 \%$ del ingreso promedio ${ }^{(37)}$

Tenemos la expectativa que la publicación de este artículo contribuya al debate sectorial e intersectorial sobre las reformas necesarias al modelo de financiamiento del sistema de salud paraguayo para lograr una protección financiera efectiva, como una cuestión fundamental para lograr la CUS.

Para responder la pregunta que nos hemos planteado, definimos como objetivo general del estudio: describir la incidencia del gasto catastrófico de salud en los hogares paraguayos y su distribución en zonas urbanas y rurales. 


\section{MATERIAL Y MÉTODOS}

El estudio fue descriptivo de corte transversal de alcance nacional. El universo fueron todos los hogares del Paraguay, siendo por tanto la unidad de análisis el hogar y no los individuos por separado.

Utilizamos la base de datos del módulo de salud de la Encuesta Permanente de Hogares (EPH) de la Dirección General de Estadística, Encuestas y Censos (DGEEC) del Paraguay del 2014. En 2015 y 2016 la EPH no recabó datos sobre gastos por motivos de salud.

La muestra fue probabilística, por conglomerados con probabilidad proporcional al tamaño, bietápico y estratificado, con representación de todo el país. Consideró distintos segmentos a partir del Censo Nacional de Población y Viviendas del 2002.

La EPH registra datos sobre los ingresos de todos los miembros del hogar. En el apartado de salud registró pagos realizados por consultas médicas, compra de medicamentos, realización de estudios diagnósticos, hospitalizaciones y otros gastos, los cuales se refieren al último evento de enfermedad o accidente en los 90 días previos a la encuesta. Los datos registrados fueron para cada miembro del hogar. Excluyó los gastos de más de un evento por persona, también los gastos habituales como los que se relacionan con el tratamiento y seguimiento de enfermedades crónicas, así como los gastos realizados por las personas que trabajan en el servicio doméstico del hogar.

Para el análisis incluimos a todos los hogares que reportaron al menos un enfermo o accidentado en el hogar.Con estos datos calculamos las siguientes variables:

-El gasto de bolsillo en salud (GBS) lo definimos como la suma de los gastos por consultas, medicamentos, estudios de diagnóstico y hospitalizaciones de todos los miembros del hogar que estuvieron enfermos o accidentados en los últimos 90 días.

- La capacidad de pago del hogar (CPH) lo definimos como la suma de los ingresos de todos los miembros del hogar descontando los gastos de subsistencia. Como la EPH recabó datos sobre el ingreso, pero no sobre gastos por alimentación ni otros, se utilizó la estructura de gastos de la DGEEC del 2012 para determinar la proporción de los ingresos que son destinados para subsistencia en cada estrato de la población estudiada. Se tomó el valor de la línea de pobreza extrema familiar como valor de gastos de subsistencia, si el valor estimado para el gasto por alimentación era superior a éste. Dado que los gastos por motivos de salud corresponden a 90 días, se calculó la $\mathrm{CPH}$ mensual y trimestral.

-El impacto posible del GBS y de sus componentes sobre la CPH lo estimamos relacionando los promedios por separado y el promedio resultante de la relación GBS/CPH para cada hogar como unidad de análisis. Calculamos también la distribución de esta relación por rangos.

- La incidencia del gasto catastrófico de salud (GCS) lo definimos como el porcentaje de todos los hogares en los cuales el GBS fue igual o superior a $10 \%, 20 \%, 30 \%$ y $40 \%$ de la $\mathrm{CPH}$. Usamos estos distintos umbrales considerando la $\mathrm{CPH}$ trimestral. Para el procesamiento de los datos utilizamos una variable dicotómica, según esta definición, considerando a los hogares que incurrieron o no en gastos catastróficos por motivos de salud.

\section{RESULTADOS}

Ell $55,8 \%$ de los hogares tuvo algún enfermo o accidentado entre sus miembros entre los cuales $81,5 \%$ tuvo algún GBS para recibir atención. Entre los hogares con GBS, 79,5\% compró medicamentos, $22,1 \%$ pagó por estudios de diagnóstico, 18,8\% lo hizo por honorarios médicos y $2,8 \%$ por hospitalizaciones (Tabla1).

EI GBS promedio de los hogares con al menos un enfermo fue de 411.627 guaraníes. Cuando consideramos sólo los que tuvieron gastos, el promedio fue de 504.807 guaraníes. El promedio de gasto por consultas fue de 125.415 guaraníes, por medicamentos 279.202 guaraníes, por estudios de diagnóstico 387.839 guaraníes y por internación 2.992.757 guaraníes. Calculamos la CPH considerando todos los ingresos y descontando los gastos de subsistencia (alimentación o línea de pobreza) y resultó en promedio 4.650.179 guaraníes mensuales para todos los hogares.

Relacionando el promedio de GBS con el promedio de la CPH mensual, se estimó el impacto posible del gasto por consultas, medicamentos, diagnóstico y hospitalizaciones (Tabla 2). 
Tabla 1: Distribución de hogares con enfermos o accidentados y GBS en los últimos 90 días. Paraguay 2014

\begin{tabular}{lll}
\hline Hogares & Número & \% sobre el total \\
\hline Total & 1.621 .525 & $100,0 \%$ \\
Hogares con enfermos & 905.320 & $55,8 \%$ \\
Hogares con GBS & 738.212 & $45,5 \%$ \\
Hogares con GBS por consultas & 170.249 & $10,5 \%$ \\
Hogares con GBS por medicamentos & 719.365 & $44,4 \%$ \\
Hogares con GBS por diagnóstico & 200.088 & $12,3 \%$ \\
Hogares con GBS por internación & 25.308 & $1,6 \%$ \\
\hline
\end{tabular}

Fuente: Elaboración propia a partir de la EPH 2014 de la DGEEC.

Tomando como unidad de análisis cada uno de los hogares calculamos el promedio de la relación GBS/CPH de un trimestre, tal como lo registra la EPH. Considerando todos los hogares el promedio resultante fue $4,6 \%$, considerando los hogares con enfermos 0 accidentados fue $8,2 \%$ y considerando sólo los que tuvieron algún GBS fue de $9,1 \%$.

Tabla 2: Impacto posible del GBS promedio sobre la CPH promedio mensual. Paraguay 2014

\begin{tabular}{ll}
\hline Concepto del gasto & GBS/CPH \\
\hline Consultas & $2,7 \%$ \\
Medicamentos & $6,0 \%$ \\
Diagnóstico & $8,3 \%$ \\
Hospitalización & $64,4 \%$ \\
GBS & $10,9 \%$ \\
\hline
\end{tabular}

Fuente: Elaboración propia a partir de la EPH 2014 de la DGEEC.

Utilizamos distintos umbrales para calcular la incidencia del GCS (GBS/CPH) en relación a todos los hogares, expresados como \% de hogares. La incidencia de GCS fue de $8,7 \%$ cuando el umbral fue GBS de $10 \%$ o más de la $\mathrm{CPH}, 4,4 \%$ cuando el umbral fue de $20 \%$, $2,8 \%$ cuando el umbral fue de $30 \%$ y $2 \%$ cuando el umbral fue de $40 \%$ (Tabla 3 ).

Tabla 3: Incidencia de gastos catastróficos de salud según distintos umbrales de la capacidad de pago de los hogares en los últimos 90 días. Paraguay 2014

\begin{tabular}{lll}
\hline Umbrales & Número de hogares & $\%$ \\
\hline $10 \%$ o más de CPH & 141.850 & 8,7 \\
$20 \%$ o más de CPH & 71.864 & 4,4 \\
$30 \%$ o más de CPH & 45.500 & 2,8 \\
$40 \%$ o más de CPH & 31.883 & 2,0 \\
\hline
\end{tabular}

Fuente: Elaboración propia a partir de la EPH 2014 de la DGEEC.

\section{DISCUSIÓN}

Varios factores pueden influir en la incidencia del GCS. En un marco analítico es razonable considerar factores dependientes de la necesidad de atención, del sistema de salud, de políticas y de las características socioeconómicas de los hogares.

Una mayor ocurrencia de enfermedades y lesiones en la población, sin mecanismos efectivos de protección financiera, tenderá a acumular mayores GBS y GCS en la población. 
También el tipo de enfermedades puede ser determinante. Por ejemplo, las enfermedades crónicas pueden generar una acumulación de gastos recurrentes en un periodo de tiempo y mayores $\operatorname{GCS}^{(20,21)}$. La gravedad también es determinante para la utilización de servicios más complejos y costosos. Cuanto más complejo es el tipo de servicio de diagnóstico o tratamiento que se requiere para la atención es esperable que se incrementen los gastos.

Cuando aumenta la accesibilidad y la utilización de servicios de salud, los gastos pueden incrementarse. Es decir, una baja incidencia de GCS puede significar tanto una buena protección financiera como una baja accesibilidad y utilización de servicios. Es posible que un grupo de hogares no experimente este tipo de gastos porque quedan excluidos del sistema de salud por no poder pagar los servicios que necesitan.

El modelo de financiamiento y de agrupación de fondos para cubrir a la población y compartir riesgos es un punto crítico de cualquier marco analítico. A menor participación del GBS como parte del gasto total de salud es esperable una menor incidencia del GCS ${ }^{(2,3)}$. El grado de cobertura de la población mediante la agrupación de fondos públicos y de seguridad social puede ser clave para reducir el GBS, pero hay que comprender que este es una parte del gasto privado de salud y que es posible que, bajo ciertas condiciones, la agrupación de fondos privados también pueda tener un efecto de reducción. Sobre este asunto hay que considerar que el sistema de salud tiene como objetivo intrínseco lograr la protección financiera, pero bajo un modelo de financiamiento equitativo. Por tanto, en un análisis amplio, los resultados sobre el grado de protección financiera (por ejemplo, en la medida que se reducen los GCS) hay que relacionarlos con el grado de equidad del sistema.

El control de precios de medicamentos, de otros productos para la salud, de tecnologías y de servicios también es determinante.

De manera indirecta las regulaciones del modelo de atención, mediante protocolos de manejo de pacientes definidos a partir de la evidencia sobre la efectividad, estándares de calidad, tecnologías más costo efectivas y otros criterios.

Políticas y programas de salud pueden priorizar tanto la atención a un grupo de enfermedades como la atención a un grupo de la población. El concepto y el alcance operativo del derecho a la salud en el sistema nacional de salud es, por tanto, determinante, sobre todo cuando éste no es sólo un enunciado, sino que además funciona mediante mecanismos concretos de garantías de cumplimiento y de exigibilidad.

Las características socioeconómicas del hogar, el nivel de ingreso, el tipo de empleo, la condición de pobreza, la educación, la tenencia de seguro, la edad de sus miembros y determinantes sociales de la salud son factores que se asocian tanto a la condición de salud como al proceso de utilización de servicios de salud ${ }^{(8)}$. Se estudia la capacidad de pago, pero también hay otros aspectos económicos que no son incluidos como el ahorro, las deudas, los planes del hogar, la capacidad de crédito y los bienes. En una sociedad donde la informalidad del empleo es alta, es pertinente considera también los costos de oportunidad, es decir lo que se deja de ganar para recibir atención ante una enfermedad o lesión.

Todos estos elementos son pertinentes para el análisis del GCS desde una perspectiva de salud pública, puesto que en este ámbito el conocimiento del problema tiene un propósito: diseñar e implementar acciones para mejorar la situación.

La EPH ofrece limitaciones y oportunidades para este tipo de análisis. Entre las limitaciones podemos mencionar las siguientes: 1) esta encuesta no está diseñada para evaluar gastos en general y particularmente no está diseñada para evaluar los gastos en salud, 2) cuando sí recaba datos sobre gastos en salud, estos no son exhaustivos, en cuanto a que no recoge información sobre todos los gastos como, por ejemplo, los gastos habituales y tampoco lo hace cuando en el periodo de 90 días las personas tuvieron más de un evento que haya motivado gastos de salud, 3) la temporalidad de 90 días permite ampliar el número de casos para el análisis, pero ésta no es coincidente con la temporalidad utilizada para otras variables como los ingresos, 4) es posible una insuficiencia de la muestra cuando se estratifican los hogares. Es aceptado que para la EPH se requieren al menos 30 observaciones válidas para establecer resultados aproximados, 5) los datos pueden ser referidos por personas que no necesariamente siempre conocen con precisión los gastos realizados por otros miembros del hogar, 6) si bien la EPH se realiza cada año, no siempre se recoge información sobre gastos en salud, por ejemplo, en 2003, 2005, 2011, 2015 y 2016 no se recabaron estos datos, 7) no es posible relacionar las necesidades de atención con los gastos, 8) no es posible relacionar la complejidad de los servicios utilizados con los gastos, y 9) no es posible determinar con exactitud la situación económica del hogar 
aunque sí se pueden obtener datos importantes como empleo, ingresos, cobertura de seguros y otros.

Pero a pesar de estas limitaciones y que los datos sobre gastos de salud no son publicados, la EPH ofrece la oportunidad de evaluar de manera periódica el comportamiento del GBS y el GCS por grandes componentes, su distribución en la población general, entre los enfermos y entre los que tuvieron que realizar pagos directos para recibir atención ante una enfermedad o accidente, ya sea desde una perspectiva de personas o desde la perspectiva de los hogares. También es posible estratificar los resultados, al menos para algunas variables, considerando distintas condiciones socioeconómicas que podría permitir construir una interpretación con enfoque de determinantes sociales de la salud.

Hemos mencionado que la OMS ha propuesto una metodología para estimar los GCS, utilizando la relación entre GBS/CPH en un periodo de tiempo y definiéndolo como $40 \%$ o más. También que este umbral puede ser distinto, de acuerdo a las características de los países y, agregamos, a las características del estudio ${ }^{(16)}$. Las publicaciones que hemos revisado señalan que esta relación es frecuentemente utilizada para determinar GCS en distintos países y contextos, pero que también se utilizan relaciones con gastos totales 0 con ingresos totales. Hemos adoptado las recomendaciones de la OMS para realizar este análisis.

Esta decisión ha implicado tres desafíos metodológicos: 1) relacionado con la determinación de la capacidad de pago, 2) relacionado con la determinación de gastos de bolsillo y 3 ) relacionado con el umbral utilizado.

Sobre la definición de la $\mathrm{CPH}$ es pertinente reiterar que la EPH no es una encuesta de ingresos y gastos. Por lo tanto, no es posible estimar la capacidad de pago de manera directa, sino que hay que recurrir a parámetros conocidos para calcularla. En este caso utilizamos la estructura de ingresos y gastos de la DGEEC del 2012. Esto nos ha permitido establecer estructuras de gastos para los hogares según distintas características y aplicarlas para realizar nuestras determinaciones. Esta definición, y procesamiento de datos, ha tenido la motivación de obtener resultados que sean comparables con estudios de otros países. Pero, las limitaciones ya señaladas y otras como las distintas definiciones de pobreza que se utilizan, hacen necesario un diseño específico, más exhaustivo, para poder realizar comparaciones válidas entre países. En nuestra opinión, si utilizamos la EPH para dar un seguimiento interno o nacional a las variaciones del GCS sería más conveniente utilizar además la relación del gasto de bolsillo con los ingresos.

Sobre la definición de GBS, la OMS propone utilizar solamente lo que hemos denominado pagos directos de los usuarios o de los miembros del hogar al momento de utilizar servicios de salud ${ }^{(16)}$. La EPH también registra otros gastos como gastos por alimentación y transporte. Estudios previos realizados en Paraguay han utilizado todos los gastos. Estos gastos, que podríamos denominar indirectos, guardan relación con la accesibilidad, sobre todo con su dimensión geográfica. En nuestra opinión, en el caso de Paraguay, con brechas de cobertura, brechas de oferta y acceso es pertinente considerar además el cálculo con todos los gastos y es esperable que cuando se desagreguen los datos estos puedan tener un mayor peso relativo en hogares rurales.

Sobre el umbral utilizado, como el GBS determinado no es exhaustivo, por los motivos ya expuestos, consideramos que los umbrales inferiores al $40 \%$ de la $\mathrm{CPH}$ son más apropiados utilizando esta fuente de datos. Concordamos que una medida razonable sería utilizar umbrales de $20 \%$ o $30 \%$. Otro elemento a tener en cuenta sobre este punto es la temporalidad de la $\mathrm{CPH}$. Nosotros hemos adoptado una estimación trimestral. En el estudio publicado por CADEP habían utilizado los ingresos trimestrales. Esto, como ya se ha señalado, debido a que los gastos se refieren a un trimestre.

Sobre la utilización de la CPH mensual o trimestral agregamos nuevos comentarios a los previos: algunas consideraciones dependientes de la unidad de análisis y otras dependientes de la variable de resultado que se estudia.

En una publicación anterior ${ }^{(37)}$ determinamos que el GBS promedio de las personas que tuvieron alguna enfermedad en 2014 fue de 245.800 guaraníes (54,5 USD) y en ésta que el GBS promedio de los hogares con al menos una persona enferma fue 411.627 guaraníes. También hemos mencionado que la EPH recoge datos sobre gastos del último evento de enfermedad o accidente por cada persona. Por lo tanto, cuando la unidad de análisis son las personas consideramos más razonable utilizar los ingresos o la capacidad de pago mensual. En cambio, cuando la unidad de análisis son los hogares consideramos más adecuado 
utilizar los ingresos o la $\mathrm{CPH}$ trimestral. Pero hay que señalar lo siguiente: en tanto la $\mathrm{CPH}$ se triplica, al pasar del análisis de las personas a los hogares, el GBS promedio se incrementa sólo en 40,3\%.

Consideramos que lo más relevante es el objetivo y consecuentemente las variables que se utilizan para determinar el GBS y el GCS. Ya hemos mencionado que desde la perspectiva de la salud pública se busca conocer los problemas para generar acciones, a través de intervenciones de políticas y programas de salud. Como ya hemos señalado, la incidencia del GCS considerando todos los hogares es un indicador que resume una información que está determinada por un conjunto de factores dependientes de la necesidad, del sistema y de las características de los hogares. Utilizando como fuente de datos la EPH y como unidad de análisis los hogares, ya señalamos que para estimar esta la incidencia de GCS consideramos más adecuado utilizar una temporalidad trimestral en todas las variables. Pero cuando se busca analizar el impacto potencial de un determinado gasto sobre la $\mathrm{CPH}$, como por ejemplo los precios promedios pagados, consideramos que es más adecuado utilizar la $\mathrm{CPH}$ mensual. Hemos utilizado esta metodología en una publicación previa, considerando como unidad de análisis a las personas y no a los hogares ${ }^{(37)}$.

Después de estas consideraciones generales, pasamos ahora a realizar la discusión específica sobre los resultados.

En primer lugar, resaltamos el alto porcentaje de los hogares con al menos 1 enfermo o accidentado y que realizaron algún tipo de GBS $55,8 \%$ y $81,5 \%$ respectivamente. Comparando estos resultados con los resultados cuya unidad de análisis fueron personas, $25,1 \%$ y $71,1 \%{ }^{(37)}$, resulta evidente que la magnitud del problema puede ser mejor comprendida desde la perspectiva de hogares. Además, es razonable sostener que, ante una enfermedad o accidente, son todos los miembros del hogar, de manera directa o indirecta, quienes dan una respuesta y apoyo o se ven afectados de alguna forma por los gastos realizados. Este hecho deriva en una cuestión práctica al momento de diseñar una política o programa de protección financiera en salud. Por ejemplo, en un programa de transferencia condicionada a familias pobres que no contempla un componente de protección financiera en salud puede resultar en que las transferencias se utilicen para pagar medicamentos o algún tipo de servicio de salud ante una enfermedad o accidente de alguno de sus miembros. Este mismo concepto puede extenderse a otros criterios de vulnerabilidad de los hogares para enfrentar gastos de salud.

En la publicación anterior, ya referida ${ }^{(37)}$, hemos señalado que el principal componente del GBS es el pago por medicamentos, seguido de estudios de diagnóstico, internaciones (u hospitalizaciones) y consultas pero que el mayor impacto potencial sobre los ingresos se debía a los gastos de hospitalizaciones ${ }^{(37)}$. Hallazgos similares habían sido publicado por CADEP utilizado la misma fuente de datos ${ }^{(32)}$. En este trabajo encontramos que el pago por medicamentos representa la mayor proporción de hogares con GBS $(44,4 \%)$ lo cual refuerza la idea de que para mitigar la participación de esta fuente de financiamiento se requiere de una política de acceso a medicamentos a nivel nacional, aunque el promedio de gasto por medicamentos tenga un impacto potencial de $6 \%$ sobre sobre la $\mathrm{CPH}$. Por otra parte, una política que pretende reducir los GCS debería priorizar mecanismos de protección financiera contra aquellas enfermedades y servicios de alto costo y que requieren de hospitalizaciones, ya que estas puede tener un impacto potencial de $64,4 \%$ sobre la $\mathrm{CPH}$, aunque afecte a una menor proporción de hogares (1,6\%).

Es sabido que el sistema nacional de salud se financia principalmente con gastos privados y sobre todo GBS $(33,34)$. Al diseñar el análisis pensamos que otra perspectiva de este modelo inequitativo podría quedar representada por las diferencias entre la relación GBS/CPH entre todos los hogares con enfermos $(55,8 \%)$ y los hogares que tuvieron gastos $(45,5 \%)$, bajo el supuesto de que a mayor diferencia mayor la inequidad del modelo de financiamiento.

Hemos señalado que la incidencia estimada de GCS utilizando la EPH es un valor inferior a la incidencia real, puesto que los datos no son exhaustivos, para cualquiera de los umbrales utilizados. Aun así, es posible identificar que el riesgo de tener GCS ante una enfermedad $\mathrm{o}$ accidente es alto y que afecta a numerosos hogares. Considerando los umbrales de $30 \%$ y $20 \%$ como los más adecuados para las características del sistema de salud paraguayo, de la población y las limitaciones de la EPH es posible que entre 45.500 y 71.864 hogares enfrenten GCS al tener que realizar pagos directos para recibir atención cuando al menos uno de sus miembros padece de alguna enfermedad o un accidente. 
Es difícil hacer comparaciones de los resultados de la incidencia del GCS estimada en este artículo para los hogares paraguayos con los resultados para otros países. Ya hemos señalado las limitaciones de la EPH para este tipo de análisis. Pero también hemos señalado que la EPH ofrece una oportunidad de evaluación y comparación interna y que este hecho permitiría el seguimiento a políticas que busquen revertir el impacto del GBS y el GCS en los hogares.

Consideramos que es pertinente desarrollar un agenda de investigación nacional sobre GBS, GCS y gastos empobrecedores. Es posible hacerlo desagregando los resultados con un enfoque de determinantes sociales de la salud. Además de estos estudios nacionales es pertinente diseñar estudios específicos para poblaciones y territorios específicos, para poblaciones vulnerables y para necesidades y servicios específicos. Finalmente, consideramos necesario establecer líneas de base y evaluar las intervenciones de políticas y programas más efectivos para controlar y reducir este tipo de gastos.

La EPH es una herramienta útil para estimar y dar seguimiento a las variaciones del GBS y el GCS, pero con limitaciones que deben considerarse al interpretar los datos. Utilizando esta fuente de datos fue posible estimar la incidencia de GCS en los hogares paraguayos. Los resultados señalan que numerosos hogares paraguayos tienen riesgo de enfrentar este tipo de gastos ante una enfermedad o accidente, sobre todo cuando se requiere de servicios de alto costo que pueden requerir hospitalizaciones. Para mitigar o controlar esta situación se requiere de políticas orientadas a tres objetivos: a) proteger a hogares vulnerables; $b$ ) brindar protección financiera ante enfermedades específicas con cobertura de servicios específicos y c) una política de alcance amplio, nacional, de acceso a medicamentos.

\section{AGRADECIMIENTOS}

A la DGEEC por su valioso aporte para la realización de este trabajo.

\section{REFERENCIAS BIBLIOGRAFICAS}

1. Frenk J. El concepto y la medición de la accesibilidad. Salud Pública de México. 1985; 27(5):438-53.

2. Organización Mundial de la Salud. La atención primaria de salud más necesaria que nunca. Informe sobre la salud en el mundo 2008. Informe de un Grupo Científico de la OMS; Ginebra: OMS; 2008. Serie de informes técnicos: clasificación NLM: W 84.6 ISBN 9789243363732.

3. Organización Mundial de la Salud. Financiación de los sistemas de salud. El camino hacia la cobertura universal. Informe sobre la salud en el mundo 2010. Informe de un Grupo Científico de la OMS; Ginebra: OMS; $2010 . \quad$ Disponible en: http://whqlibdoc.who.int/whr/2010/9789243 564029_spa.pd

4. Murray CJ, Frenk J. Sistemas de Salud: Un marco para evaluar el desempeño de los Sistemas de Salud. OMS. 2000; Recopilación de artículos número 3: 149 162.http://whqlibdoc.who.int/boletin/2000/R A_2000_3_149-162_spa.pdf

5. Tobar F. Políticas de Salud. Modalidades y Tendencias. Programa de Investigación Aplicada. Programa de Investigación Aplicada. Buenos Aires: Instituto Universitario ISALUD, 2000.

6. Kruk M, Porignon D, Rockers PC, Van Lerberghe $W$. The contribution of primary care to health and health systems in lowand middle-income countries: A critical review of major primary care initiatives, Social Science \& Medicine 2010; 70(6): 904-11.

7. https://EconPapers.repec.org/RePEc:eee:s ocmed:v:70:y:2010:i:6:p:904-911.

8. Lewin S, Lavis JN, Oxman AD, Bastías G, Chopra M, Treweek $S$, et al. Supporting the delivery of cost-effective interventions in primary health-care systems in low-income and middle-income countries: an overview of systematic reviews. Lancet 2008; 372(9642): 928-39.

9. Organización Mundial de la Salud. Reducir las inequidades sanitarias actuando sobre los determinantes sociales de la salud. $62^{a}$ Asamblea Mundial de la Salud. Disponible en: http://apps.who.int/gb/ebwha/pdf_file s/A62/A62_R14-sp.pdf

10. OPAS. Renovação da Atenção Primária em Saúde nas Américas: documento de posicionamento da Organização PanAmericana da Saúde/Organização Mundial da Saúde (OPAS/OMS). Washington, DC: OPAS; 2007.

11. OPS. Redes Integradas de Servicios de Salud: Conceptos, Opciones de Política y Hoja de Ruta para su Implementación en las Américas" Washington, D.C.: OPS, (C) 2010.

12. Organización Mundial de la Salud. Informe sobre la salud del mundo 2013: investigaciones para una cobertura sanitaria universal. Ginebra, Suiza. OMS; 2013.

13. Organización Mundial de la Salud. Declaración de Adelaida sobre la Salud en Todas las Políticas: hacia una gobernanza compartida en pro de la salud y el bienestar. Adelaida, 2010. 
14. WHO. Health in 2015: from MDGs, Millennium Development Goals to SDGs, Sustainable Development Goals.Ginebra, 2015.

15. Chan M. Alocución ante la 65 Asamblea Mundial de la Salud. Disponible en: http://apps.who.int/gb/ebwha/pdf_files/WHA65/A65_3-sp.pdf

16. Ministerio de Salud Pública y Bienestar Social y OPS. Cuentas Nacionales de Salud. Asunción Paraguay. 2003 Disponible en: http://www.paho.org/par/index.php?option =com_docman\&view=download\&category_ slug =publicaciones-concontrapartes\&alias $=64$-cuentas-de-saludparaguay-octubre-2003\&Itemid $=253$

17. Xu K. Organización Mundial de la Salud. Distribución del Gasto en Salud y gastos catastroficos. Metodologia; Informe de un Grupo Científico de la OMS; Ginebra: OMS; 2005.

18. Xu K, Evans DB, Kawabata K, Zeramdini R, Klavus J, Murray CJL. Household catastrophic health expenditure: a multicountry analysis. Lancet. 2003;362:111-7.

http://www.who.int/entity/health_financin g/documents/lancet-

catastrophic_expenditure.pdf, accessed 06 May 2015

19. Abeldaño RA. Analysis of household expenditure on healthcare in Argentina, as a component of universal health coverage. Ciência\&SaúdeColetiva, 2017; 22(5):163140.

20. Barros Aluísio JD, Bastos João L, Dâmaso $\mathrm{AH}$. Catastrophic spending on health care in Brazil: private health insurance does not seem to be the solution. Cad. SaúdePública [Internet]. 2011 [cited 2017 July 26]; 27(Suppl 2): s254-s262. Available from: http://www.scielo.br/scielo.php?script=sci_ arttext\&pid=S0102-

$311 \times 2011001400012 \&$ Ing $=$ en. $\quad$ Doi: 10.1590/S0102-311X2011001400012.

21. Liu X, Sun X, Zhao $Y$, Meng Q. Financial protection of rural health insurance for patients with hypertension and diabetes: repeated cross-sectional surveys in rural China. BMC Health Services Research 2016; 16:481.

22. Wang Z, Li X, Chen M. Catastrophic health expenditures and its inequality in elderly households with chronic disease patients in China. Int J Equity Health. 2015;14(1):8

23. Sesma-Vázquez $S$, Pérez-Rico $R$, \& Lino Sosa-Manzano C. Gastos catastróficos por motivos de salud en México: magnitud, distribución y determinantes. Salud Pública de México, 2015. Recuperado de http://saludpublica.mx/index.php/spm/arti cle/view/4687/4535

24. Weid T, Emmert M, Schöffski O. El impacto de los gastos catastróficos como amenaza existencial para las familias no asalariadas en el área metropolitana de la ciudad de
México. Aten Fam. octubre de 2014;21(4):103-8.

25. Knaul FM, Arreola-Ornelas H, MéndezCarniado O. Financial protection in health: updates for Mexico to 2014. Salud Publica Mex 2016; 58: 341-50. Doi: 10.21149/spm.v58i3.7886

26. Pérez G, Silva A. Una mirada a los gastos de bolsillo en salud para Colombia. Revista del Banco de la República. 2015;27-55.

27. Perticara M. Incidencia de los gastos de bolsillo en salud en siete países latinoamericanos. Santiago de Chile: CEPAL División de Desarrollo Social; 2008. $68 p$.

28. Castillo Laborde C, Villalobos P. Caracterización del gasto de bolsillo en salud en Chile: una mirada a dos sistemas de protección. RevMed Chile 2013; 141: 1456.

29. Díaz-González E, Ramírez-García JB. Gastos catastróficos en salud, transferencias gubernamentales y remesas en México. Papeles de Población. 2017; 23(91). Universidad Autónoma del Estado de México. Disponible en: http://www.redalyc.org/articulo.oa?id=112 50327008

30. Bernal N, Carpio MA, Klein TJ. The effects of access to health insurance: evidence from a regression discontinuity design in Peru. J Public Econ. 2017;154:122-36.

31. Particara M. Incidencia de los gastos de bolsillo en salud en siete países latinoamericanos. Santiago de Chile: CEPAL Serie Políticas Sociales N 141. ISSN versión electrónica 1680-8983. 2008.

32. Díaz-González E, Ramírez-García JB. Gastos catastróficos en salud, transferencias gubernamentales y remesas en México. Papeles de Población, 2017; 23(91). Universidad Autónoma del Estado de México. Disponible en: http://www.redalyc.org/articulo.oa?id=112 50327008

33. Benítez G. Paraguay: Distribución del Gasto en Salud y Gastos de Bolsillo. Principales resultados. CADEP. Asunción Paraguay. 2017. Disponible en: http://www.cadep.org.py/uploads/2017/06 /Gastos-de-bolsillo-en-salud-OK.pdf

34. Rodríguez JC, Villalba R. Gasto y gestión de la salud pública. Situación y escenario materno-infantil. Investigación para el Desarrollo. Proyecto "Debates y acciones para mejorar la calidad de la inversión social en Paraguay". ISBN: 978-99967811-6-2 Asunción Paraguay. 2016. Disponible

en: http://www.desarrollo.org.py/admin/app/w ebroot/pdf/publications/04-08-2016-1009-56-1820147387.pdf

35. Dalal K. Evolution and patterns of global health financing 1995-2014: development assistance for health, and government, prepaid private, and out-of-pocket health 
spending in 184 countries. TheLancet 2017; 6736(17)30874-7. Doi: $10.1016 / S 0140$.

36. Gaete R. Financiamiento y Gasto en Salud. Fundación CIRD. 2017. Disponible en: https://es.slideshare.net/rubengaete3/fina nciamiento-y-gasto-en-salud-en-elparaguay

37. DGEEC. Principales resultados de la Encuesta Permanente de Hogares 2015.
Disponible en: http://www.dgeec.gov.py/Publicaciones/Bi blioteca/resultado\%20eph2015/PUBLICACI ONES\%20EPH\%202015\%2008-05-17.pdf

38. Giménez Caballero $\mathrm{E}$, Rodríguez JC, Ocampos G, Flores L. Composición del gasto de bolsillo en el sistema de salud del Paraguay. MemInstInvestigCienc Salud. 2017; 15(3): 64-72. 